\title{
Immunohistological Localization of Mella Melatonin Receptor in Pigeon Retina
}

This article was published in the following Dove Press journal:

Nature and Science of Sleep

\author{
Wenlong Sheng' \\ Shijun Weng ${ }^{2}$ \\ Fei Li' \\ Yun Zhang' \\ Qiuxia $\mathrm{He}^{\mathrm{I}}$ \\ Wenxiang Sheng' \\ Ying $\mathrm{Fu}^{3}$ \\ Haiyue Yan $^{4}$ \\ Kechun Liu' \\ 'Biology Institute, Qilu University of \\ Technology (Shandong Academy of \\ Sciences), Jinan, People's Republic of China; \\ ${ }^{2}$ State Key Laboratory of Medical \\ Neurobiology, Institutes of Brain Science, \\ Department of Ophthalmology, Zhongshan \\ Hospital, Fudan University, Shanghai, \\ People's Republic of China; ${ }^{3}$ Shandong \\ Science and Technology Exchange Center, \\ Jinan, People's Republic of China; \\ ${ }^{4}$ Shandong Institute of Scientific and \\ Technical Information, Jinan, People's \\ Republic of China
}

Background: Melatonin (N-acetyl-5-methoxytryptamine), a significant indoleamine neuromodulator implicated in circadian rhythms and sleep patterns, regulates diverse rhythmic functions via activating its high-affinity G-protein-coupled receptors. However, the detailed cellular expression of the Mella receptor in the retina is still a research gap.

Methods: The expression of the Mella receptor in pigeon retina was assessed using Western blot analysis and immunofluorescent staining. The cellular localization of the Mella receptor was studied using double immunofluorescent staining and laser-scanning confocal microscopy.

Results: Our data suggested that the Mella receptor was extensively expressed in the outer segment of Rho4D2-labeled rod and L/M-opsin-labeled red/green cone and in the somata of the CB-labeled horizontal cell, TH-labeled dopaminergic amacrine cell, ChAT-labeled cholinergic amacrine cell, PV-labeled AII amacrine cell, Brn3a-labeled conventional ganglion cell, melanopsin-containing ganglion cell and CRALBP-labeled Müller glial cell. In addition, the Mella receptor was diffusely distributed throughout the full thickness of the inner plexiform layer. However, the outer segment of S-opsin-labeled blue cone, the somata of ChX-10-labeled bipolar cell and outer plexiform layer seemed to lack immunoreactivity of the Mella receptor.

Conclusion: The finding that multiple types of retinal cells express the Mella receptor provides a new neurobiological basis for the participation of melatonin in the regulation of retinal functions through activating the Mella receptor.

Keywords: circadian rhythm, retinal Mella receptor, cellular localization, diurnal animal

\section{Introduction}

Neuromodulator melatonin, serving as a neurohormonal message of the photoperiod, is implicated in numerous biological activities, and in non-mammalian vertebrates it owns high-affinity G-protein-coupled receptors Mella (named $\mathrm{MT}_{1}$ in mammalian), Mellb (named $\mathrm{MT}_{2}$ in mammalian), and Mellc. Melatonin is produced and secreted in a daily rhythmic manner with the maximal concentration always occurring during the dark phase, ${ }^{1}$ and modulates numerous retinal processes, such as membrane conductance, ${ }^{2}$ resting potentials, ${ }^{3}$ multiple types of membrane channels ${ }^{4}$ and synaptic transmission. ${ }^{4}$ These actions of melatonin or melatonin receptors indicate the melatonin system is indeed implicated in many retinal functions. Functionally, retinal melatonin is involved in the modulation of various physiological responses, including rod disc shedding, dopamine release, visual sensitivity, ${ }^{5}$ retinal rhythmicity, ${ }^{6}$ and sleep. ${ }^{1}$
Correspondence: Wenlong Sheng; Kechun Liu

Email shengwenlongII21@163.com; liukechun2000@163.com 
Previous studies show that the localization of melatonin receptors appears enormously species-dependent and/ or cell subtype-dependent. This might help to explain why the biological rhythm is abundant and various in diurnal animals and nocturnal animals. A number of studies related to the expression of retinal melatonin receptors have been carried out in nocturnal animals, such as the mouse and rat. ${ }^{6-9}$ However, the localization of the melatonin receptor in diurnal animals is less investigated. In humans, melatonin exerts its neuromodulation via its specific receptors. ${ }^{10,11}$ In a description of morphology on mRNA transcription these receptors are widely located in chick retina. ${ }^{12}$ In fact, they do not fully identify the type of neuron or glial cell which expresses Mella, Mellb, and Mel1c receptors in the retina. Our recent work shows that multiple types of retinal cell, including Rho4D2-labeled rod, L/M-opsin-labeled red/green cone, S-opsin-labeled blue cone, TH-labeled dopaminergic amacrine cell (AC), ChAT-labeled cholinergic AC, PV-labeled glycinergic AII AC, Brn3a-labeled conventional ganglion cell (GC), melanopsin-containing GC express Mel1b receptor in pigeon retina, while CB-labeled horizontal cell (HC), ChX-10labeled bipolar cell (BC), and CRALBP-labeled Müller glial cell seem to lack Mellb receptor immunoreactivity. ${ }^{8}$ However, the detailed cellular expression of the Mella receptor in the retina is still a research gap. Here, we investigate the cellular localization of melatonin receptor Mel1a in pigeon retina. Our findings indicate that the Mel1a receptor is widely and selectively expressed in retinal neurons and glial cells as well as inner plexiform layer (IPL). These results suggest melatonin in the retina may directly modulate the diversified activities of retinal neurons and glial cells by modulating melatonin receptor Mella.

\section{Materials and Methods}

\section{Animals and Ethics Statement}

A total of nine adult pigeons (Columba livia domestica) were recruited in the present study. Adult pigeons were housed for at least 3 weeks in a 14 hour light ( 1,200 lux): 10 hour dark (LD) cycle before sacrifice. Additionally, to reduce possible diurnal factors on the level of the Mella receptor expression, all tissues were collected at zeitgeber time 6-8 (ZT6-8). Use and handling of animals were strictly in accordance with the Institutional Animal Care and Use Committees of Qilu University of Technology whose guidelines were based on the US National
Institutes of Health (NIH). Ethical approval was granted by the Institutional Animal Care and Use Committees of Qilu University of Technology.

\section{Tissue Preparation}

Experimental birds were euthanized with urethane overdose and enucleated. Eyecups were rapidly isolated in Hank's balanced salt solution and transferred into freshly prepared 4\% PFA in ice-cold 0.01 M PBS (pH 7.5) for 0.5 hour. Then tissues were emerged in $30 \%$ sucrose in PBS in a refrigerator for dehydration, and eyecups were embedded in embedding agent. A CM1950 cryostat was selected to cut $20 \mu \mathrm{m}$ slices, and slices were finally fixed to microscope slides.

\section{Antibodies}

A new but widely-used polyclonal antibody ${ }^{6,7}$ designed against a peptide corresponding to a region of the $3 \mathrm{rd}$ intracellular loop of the mouse $\mathrm{MT}_{1}$ receptor (residues 223-236 (C) RVKPDNKPKLKPQD) was generated in rabbits (AMR-031, 1:200 dilution, Alomone laboratories, Jerusalem, Israel) for immunohistochemical staining of the Mella receptor. For double labeling, the sections were incubated sequentially in a mixture of two kinds of primary or secondary antibody mentioned in our previous study. ${ }^{8}$ Mouse anti-Rho4D2 antibody (1:1,500 dilution, ab98887, Abcam plc., UK), goat anti-S-opsin antibody (1:500 dilution, sc-14363, Santa Cruz Bio-technology, USA) and goat anti-L/M-opsin antibody (1:800 dilution, sc-22117, Santa Cruz Bio-technology, USA) were separately used for labeling rods, blue cones, and red/green cones. Mouse anti-calbindin D-28k (CB) monoclonal antibody (1:2,000 dilution, 300, Swant, Switzerland) and sheep anti-homeobox protein ChX10 polyclonal antibody (1:400 dilution, ab16141, Abcam plc., UK) were respectively used for labeling HCs and BCs. Antibodies used to mark different subtypes of ACs were as follows: goat anticholine acetyltransferase (ChAT) polyclonal antibody (1:800 dilution, AB144P, Chemicon, USA) for cholinergic ACs, mouse anti-tyrosine hydroxylase (TH) monoclonal antibody (1:5,000 dilution, T1299, Sigma, USA) for dopaminergic ACs, and mouse anti-parvalbumin (PV) monoclonal antibody (1:1,000 dilution, 235, Swant, Switzerland) for glycinergic AII ACs. Goat anti-Brn3a polyclonal antibody (1:800 dilution, sc-31984, Santa Cruz Bio-technology, USA) was used for labeling GCs and mouse anti-melanopsin monoclonal antibody (1:200 dilution, sc-515838, Santa Cruz Bio-technology, USA) 
was used for labeling ipRGCs. Mouse anti-cellular retinaldehyde-binding protein (CRALBP) monoclonal antibody (1:1,000 dilution, ab15051, Abcam plc., UK) was used for labeling Müller cells.

Secondary antibodies used in this work were as follows: Alexa Fluor 488-linked donkey anti-rabbit IgG for labeling Mella receptor; Alexa Fluor 555-linked donkey anti-mouse IgG for Rho4D2, CB, TH, PV, melanopsin, and CRALBP; Alexa Fluor 555-linked donkey anti-goat IgG for S-opsin, L/M-opsin, ChAT, and Brn3a; Alexa Fluor 555-linked donkey anti-sheep IgG for ChX10 (all 1:200 dilution, Invitrogen, USA). Horseradish peroxidase-conjugated donkey anti-rabbit IgG (1:2,000, Santa Cruz Biotechnology, USA) was used for labeling Mel1a receptor in Western blot.

\section{Immunohistochemistry on Retinal Slices}

Experimental procedures were performed according to the method described previously ${ }^{8}$ with minor modifications. Briefly, tissues were pretreated in blocking solution for 2 hours to block non-specific bindings, and then treated with the Mella receptor antibody in an incubating medium at $4^{\circ}$ $\mathrm{C}$ for 72 hours. Then the tissues were treated with donkey anti-rabbit IgG antibody for 2 hours. In control trials, the total operation was coincident except that the tissues were treated with a solution containing the primary antibody and the relevant antigen peptide. All fluorescent labeling trials were conducted on more than four pigeons.

\section{Confocal Laser Scanning Microscopy and Data Analysis}

A Fluoview FV1000 confocal laser scanning biological microscope (Olympus Corporation, Tokyo, Japan) was used to view the sections and acquire the images at $40 \times$ magnification. Images were resized and adjusted for global brightness and contrast using the software Photoshop CS3 (Adobe Systems, San Jose, CA, USA).

\section{Western Blot}

The concentration of protein from the retinas was determined using a BCA Protein Assay (Thermo Scientific). Extracted supernatants $(20 \mu \mathrm{g} / \mathrm{lane})$ were electrophoresed on $10 \%$ sodium dodecyl sulfate-polyacrylamide gel and electro-blotted onto nitrocellulose (NC) membranes. The membranes were then treated with block solution for 2 hours and dealt with the diluted Mella receptor antibody for 2 hours at room temperature and 12 hours at $4^{\circ} \mathrm{C}$, and then followed by being incubated in HRP-conjugated secondary antibody (1:4,000 dilution, Millipore Corp., Billerica, MA, USA) for 2 hours. Specific binding was developed via Western blotting substrate and digital images were taken using ChemiDoc XRS System with Image Lab software (Bio-Rad, Hercules, CA, USA). In load control trials, the $\mathrm{NC}$ membranes were treated with the solution containing anti-Mella receptor antibody and the relevant immunizing peptide.

\section{Results \\ Profile of Mella Receptor in Pigeon Retina}

In the retinal supernatants from the birds, the Mella receptor antibody revealed a predominant band about 40 $\mathrm{kDa}$ (Figure 1A), which was consistent with the early report. ${ }^{13}$ No obvious band was detected if the antibody was pre-treated with the relevant immunizing peptide (Figure 1B). These results suggested the protein identified by our antibody might well be a Mella receptor.

In the retinal section (Figure 1C) Mella receptor immunoreactivity was detectable in the outer photoreceptor layer, corresponding to the outer segment (OS) of photoreceptors. Mella receptor-immunoreactivity clearly existed in many cells of the inner nuclear layer (INL) and they could be inhibitory neurons (such as HCs and ACs), excitability interneurons (such as BCs), output neurons (such as displaced GCs), and/or glia cells (such as Müller cells). Moreover, signals for the Mella receptor were strong in the ganglion cell layer (GCL), which might be displaced ACs (like cholinergic ACs) and/or GCs. In addition, very dispersive labeling for the Mella receptor was diffusely distributed throughout the full thickness of the IPL, while no clearly Mella receptor signal was visible in the outer plexiform layer (OPL). Note that the staining of the Mella receptor could be completely eliminated with the corresponding immunogen peptide (Figure 1D). Figure 1E describes the retinal layers definitely.

\section{Expression of Mella Receptor in Outer Photoreceptors}

To study the expression of melatonin receptor Mella in the outer photoreceptors, we performed double labeling studies. Figure 2A-C shows localization of the Mella receptor in rods. Double immunolabeling revealed Rho4D2labeled OS of the rod observed in seven vertical sections 

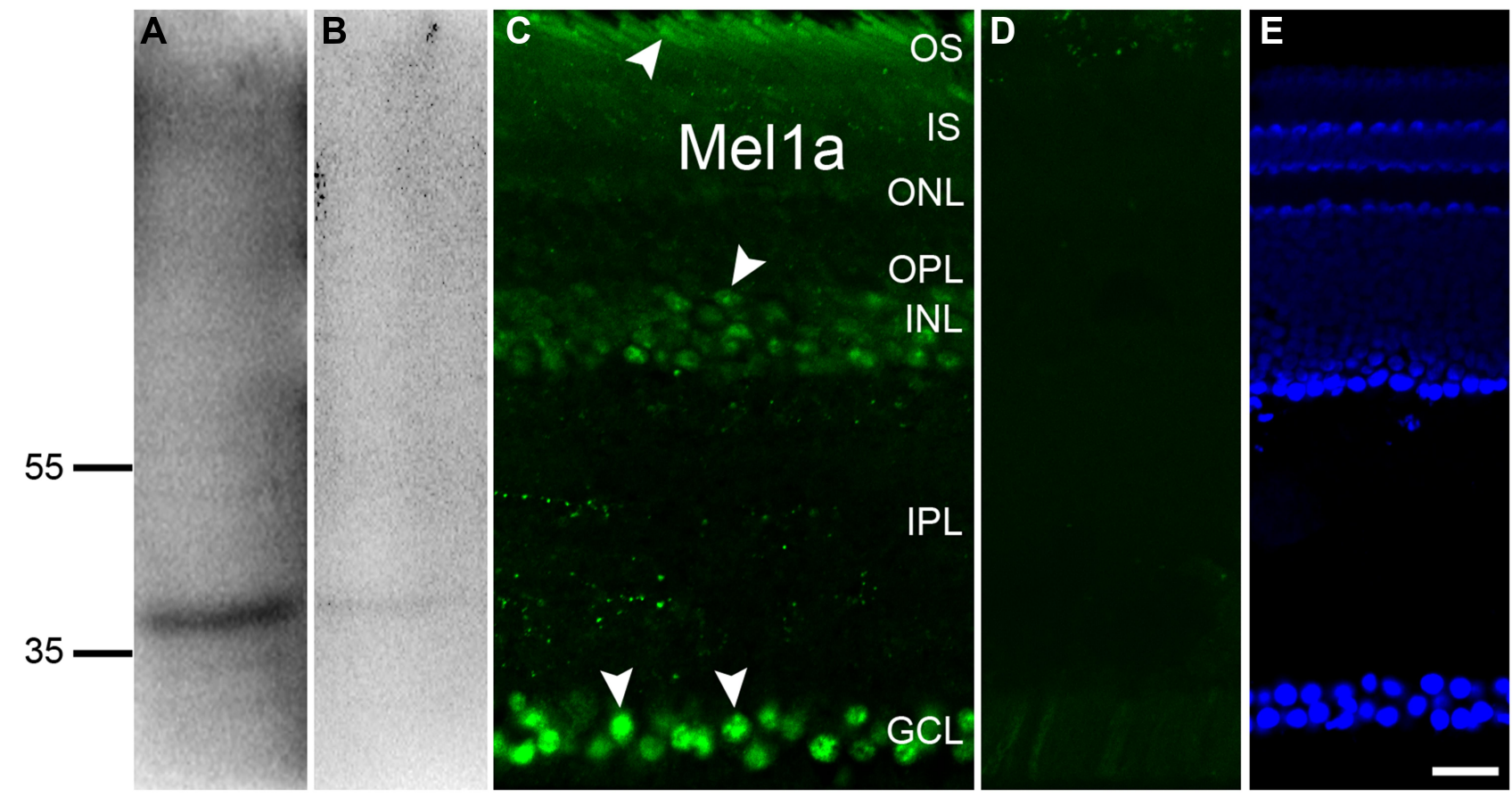

Figure I Profile of Mella receptor in the retina. (A) Qualitative analysis of Mella receptor protein in pigeon tissue reveals an expected signal at $40 \mathrm{kDa}$. (B) After the antibody being incubated with immunogen peptide, such a band is no longer observed. (C) Immunofluorescence staining of a vertical slice with anti-Mella (arrowhead) antibody. (D) Micrograph of a retinal slice in the negative control shows no clear signal is detectable if the primary antibody is treated with the corresponding antigen peptide in advance. (E) DAPI-indicated nuclei reveal the hierarchical structure of vertical slice. Representative neurons and glia are marked by white arrowheads. Scale bar $=20 \mu \mathrm{m}$, the same below.

were strongly labeled by the Mella receptor antibody with a $100 \%$ positive rate (356 out of 356 cells). Figure $2 \mathrm{D}-\mathrm{F}$ shows localization of the Mella receptor in blue cones. Double immunolabeling revealed that the Mella receptor immunoreactivity was undetectable on the S-opsin-labeled OS of the blue cone (133 out of 133 cells from six sections, $0 \%$ positive rate). Figure $2 \mathrm{G}-\mathrm{I}$ shows localization of the Mella receptor in red/green cones. Double immunolabeling shows the Mella receptor signal was strong on the OS of L/M-opsin-labeled red/green cone (284 out of 286 cells from six sections, $99 \%$ positive rate), but not on the inner segment (IS).

\section{Expression of Mella Receptor in the HCs and BCs}

We made more investigations to explore the localization of Mella receptor in $\mathrm{HCs}$ and BCs. Figure $3 \mathrm{~A}-\mathrm{C}$ shows the localization of the Mella receptor in HCs. CB-immunolabeled HCs characterized by large somata were situated in the distal edge of the INL. Double immunolabeling indicated that the CB-labeled HCs were clearly Mella receptor positive. A total of $25 \mathrm{CB}$-labeled neurons in 15 retinal slices were identified in the outer INL, and they were co- labeled by Mel1a receptor (25 out of 25 cells, $100 \%$ positive rate). It was noteworthy that the processes of these cells were negative for Mella receptor. Figure 3D$\mathrm{F}$ shows the localization of the Mella receptor in BCs. Double immunolabeling displayed that the somata of the BCs were not labeled by Mella receptor (Figure 3F). Our results demonstrated $33 \mathrm{ChX} 10$-positive neurons in four retinal slices appeared to be Mella receptor negative ( 0 out of 33 cells, $0 \%$ positive rate).

\section{Expression of Mella Receptor in the ACs}

To explore the expression of the Mella receptor in ACs, we performed fluorescent labeling studies using the Mella receptor antibody together with specific and reliable markers for different types of ACs. Dopaminergic neurons solely existed in the innermost layer of the INL, while their dendrites were mostly located in the outermost layer of the IPL. Fluorescent immunolabeling suggested that TH-labeled dopaminergic neurons were found to be Mella receptor immunoreactivity (Figure 4A-C). Thirty-two cells with obvious $\mathrm{TH}$ immunostaining were observed in 25 retinal slices, and they were mostly Mel1a receptor-positive (31 out of 32 cells, 97\% positive rate). Simultaneously, the processes of the TH-positive cells were not clearly labeled 


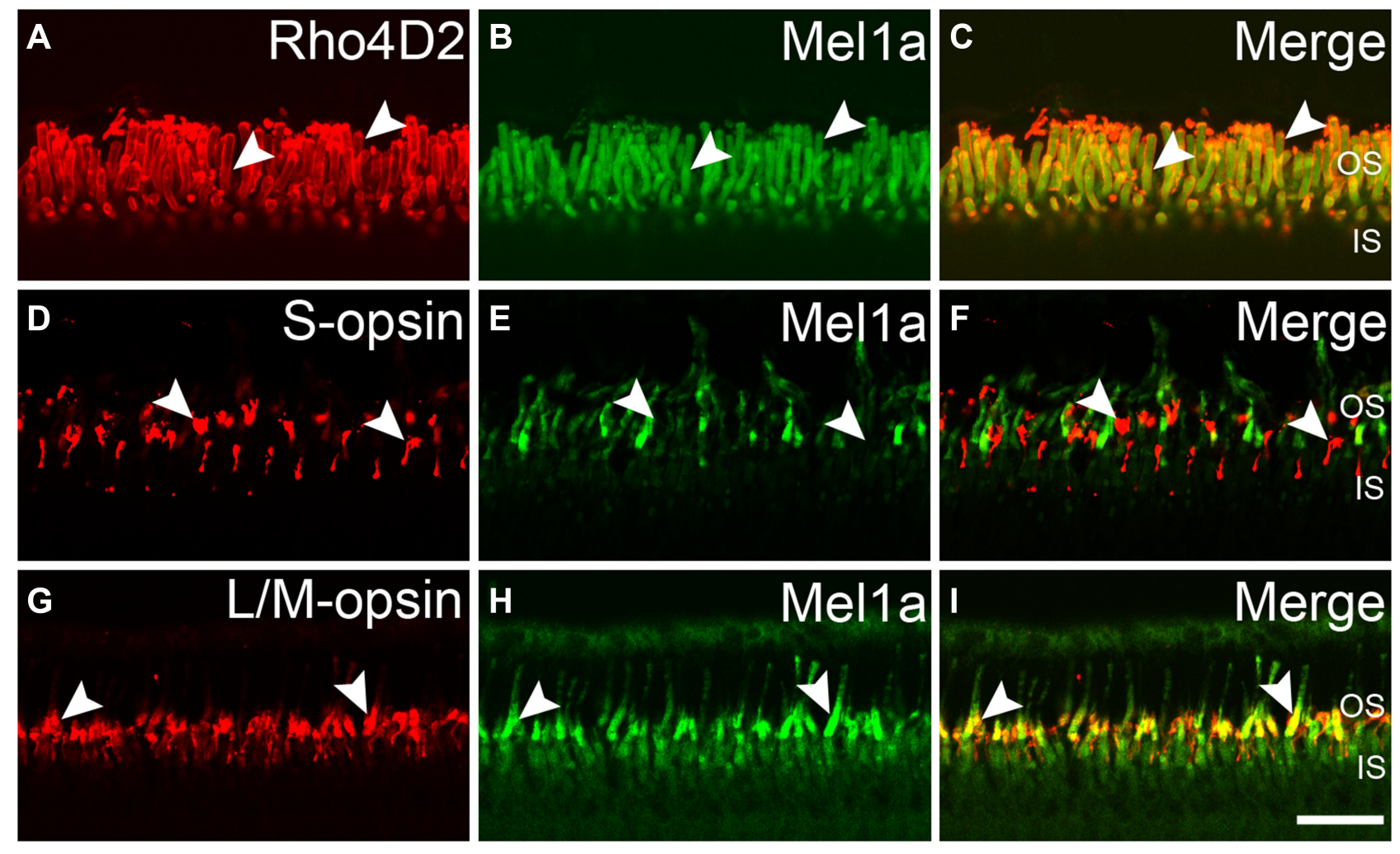

Figure 2 Mella receptor immunoreactivity in outer photoreceptors. (A-C) Representative data from rod-rich retinal slice double-labeled by Rho4D2 and Mel la receptor antibodies. Mella receptor signal is observed in the OS of Rho4D2-positive photoreceptors clearly. (D-F) Representative data of a cone-dominant retinal slice doublelabeled by S-opsin and Mella receptor antibodies. The OS of S-opsin-positive blue cone photoreceptors are Mella receptor-negative. (G-I) Representative data of a conedominant retinal slice double-labeled by L/M-opsin and Mella receptor antibodies. Note that yellowish appears in the OS of L/M-opsin-labeled red/green cone photoreceptors. Representative neurons are marked by white arrowheads.
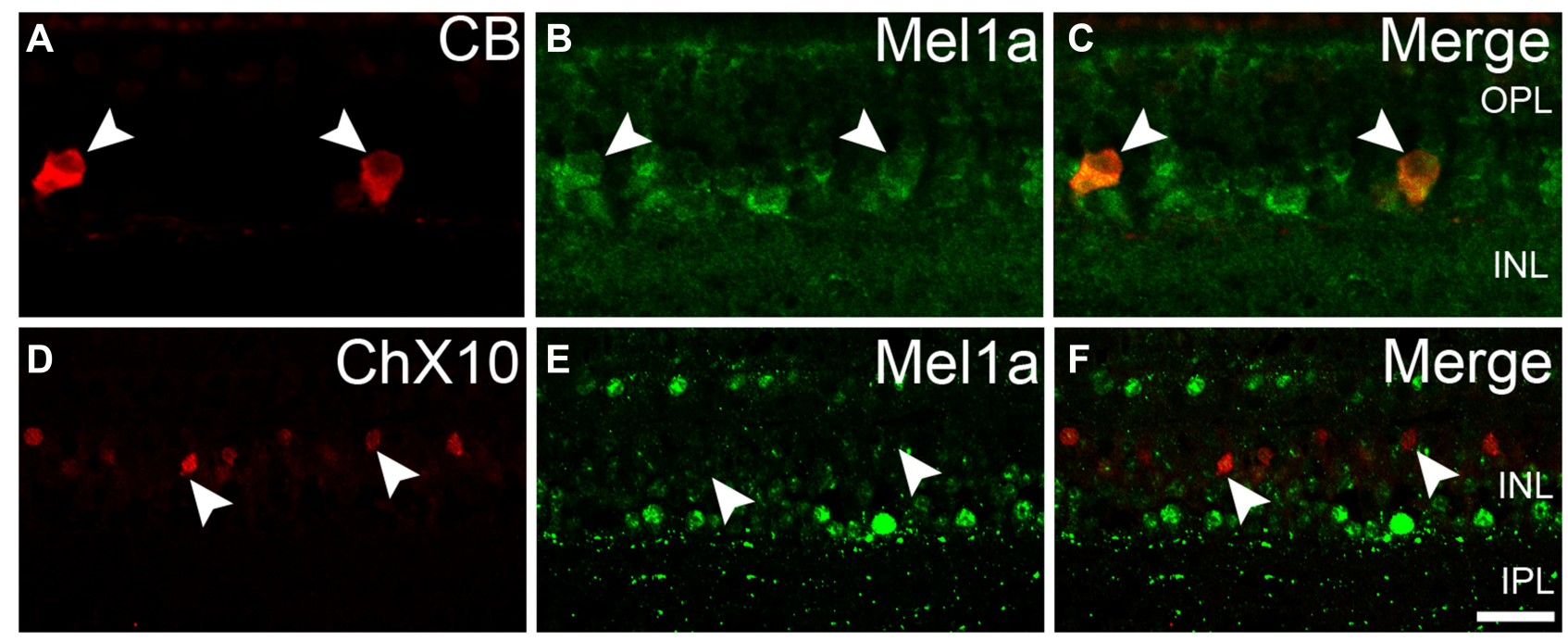

Figure 3 Mella receptor immunoreactivity in $\mathrm{HCs}$ and BCs. (A-C) Representative data from retinal slice double-labeled by CB and Mella receptor antibodies. The somata of CB-positive neurons are co-labeled by Mella receptor. (D-F) Representative data of a retinal slice double-labeled by ChXIO and Mella receptor. All ChXI0-labeled neurons are Mella receptor-negative. Representative neurons are marked by white arrowheads.

by Mella receptor. The cholinergic neurons were located in both the GCL and INL and mirror-symmetrically arranged, while their dendrites constituted two obvious braids in the
IPL. Double labeling in Figure 4D-F showed ChAT-immunoreactive cholinergic ACs were also Mella receptor immunoreactive, while no clearly Mella receptor 


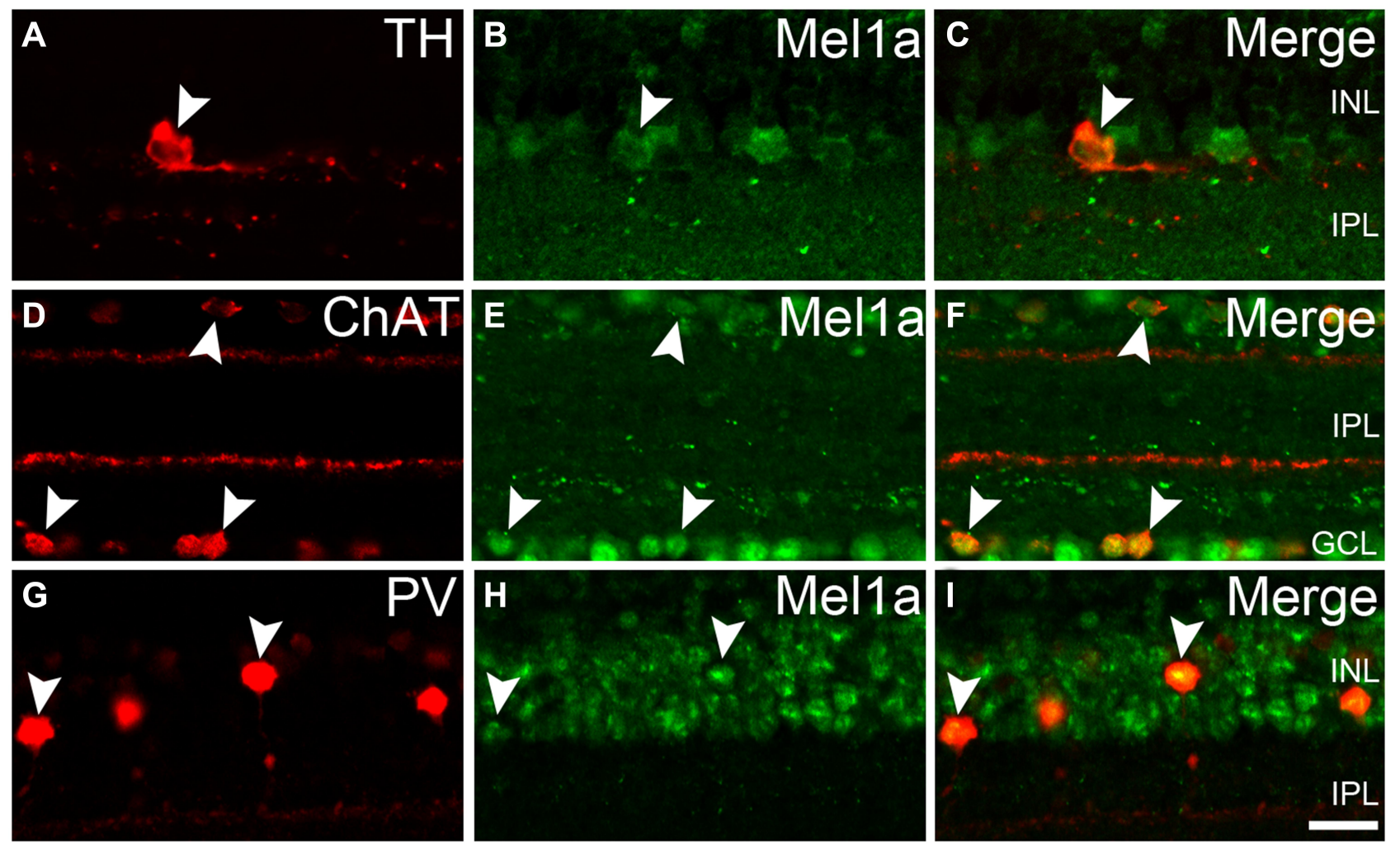

Figure 4 Mel la receptor immunoreactivity in several subpopulations of retinal ACs. (A-C) Representative data from retinal slice double-labeled by TH and Mel la receptor. TH-positive dopaminergic neuron is clearly labeled by Mella receptor. (D-F) Representative data of a retinal slice double-labeled by ChAT and Mella receptor. Mirrorsymmetrically arranged ChAT-positive neurons express Mella receptor obviously. (G-I) Representative data of a retinal slice double-labeled by PV and Mella receptor. PVpositive glycinergic neurons are clearly Mel la receptor-positive. Representative neurons are marked by white arrowheads.

immunoreactivity was found in the two braids. In a quantitative analysis involving 119 ChAT-positive neurons collected from 14 retinal slices, none of these ChAT-positive
ACs were not Mella immunoreactive. PV-positive AII neuron, characterized by these processes ended at distinct layers of the IPL was the mainly well-known subpopulation of
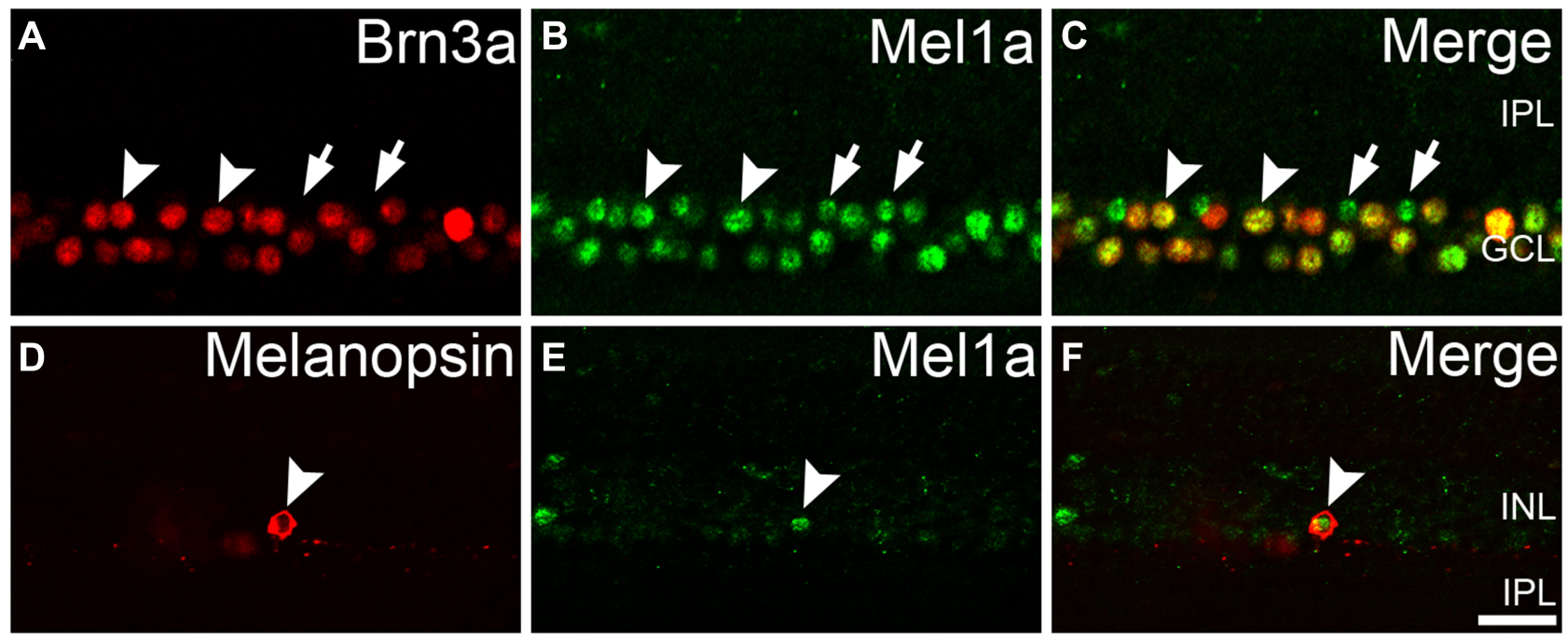

Figure 5 Mell a receptor immunoreactivity in retinal GCs. (A-C) Representative data from retinal slice double-labeled by Brn3a and Mell a receptor. Clear Mel la receptor immunostaining is observed in all Brn3a-positive GCs. Some Mel la receptor-positive cells (locations marked by the arrows) in the GCL are not immunoreactive for Brn3a, and they may be displaced ACs (cholinergic neurons in Figure 4D were typical examples) or Brn3a-negative GCs (such as inner photoreceptor ipRGC). (D-F) Representative data of a retinal slice double-labeled by melanopsin and Mella receptor. Note that Mella receptor immunostaining is observed in soma of melanopsincontaining photoreceptor. Representative neurons are marked by white arrowheads and arrows. 
glycinergic neuron. Double-labeling studies unambiguously indicated that Mella receptor obviously existed in AII ACs, while their dendrites were not marked by Mella receptor prominently (Figure 4G-I). Our results demonstrated that all the PV-immunolabeled AII neurons observed in this study were co-labeled by Mel1a receptor (38 out of 38 cells from four retinal slices with the positive rate being 100\%).

\section{Expression of Mella Receptor in the GCs}

The Mella receptor-immunoreactive neurons situated in the innermost cell layer might be ACs and/or GCs. More experiments were conducted to investigate whether the Mella receptor existed in GCs (Figure 5). Our results demonstrated that 432 GCs with strong immunofluorescence signal for Brn3a were observed in 16 retinal slices, and all of them were Mellapositive (100\% positive rate). The ipRGC was a kind of Brn3a-negative and melanopsin-positive GC. Immunofluorescence labeling experiment in 14 retinal slices indicated the Mella receptor was situated in melanopsin-containing ipRGC (13 out of 13 cells with a $100 \%$ positive rate, Figure 5D-F).

\section{Expression of Mella Receptor in the Müller Cells}

The retinal glia Müller cells, the main gliocyte, can be labeled by CRALBP. Double-immunolabeling experiment (Figure $6 \mathrm{~A}-\mathrm{C}$ ) was performed for assessing the expression of Mella receptor in CRALBP-labeled Müller cell. Intense staining for the Mella receptor was detected in the somata of Müller cells
(100 out of 101 cells from 15 retinal slices, with a 99\% positive rate), while no obvious Mella receptor-immunoreactivity was visible in the inner and outer stem processes.

\section{Discussion}

Melatonin, eliciting its biological functions with Mella and Mel1b receptors (corresponding to mammalian $\mathrm{MT}_{1}$ and $\mathrm{MT}_{2}$ receptors, respectively), is a significant neuromodulator in circadian rhythm systems. ${ }^{1,14,15}$ Numerous research studies have mainly stayed at the retina of rat, mouse, and others, which belong to nocturnal animals. In the adult rat retina, the melatonin receptor $\mathrm{MT}_{1}$ immunocytochemically locates in the IPL, OPL, and ipRGCs, ${ }^{1,6,7}$ while $\mathrm{MT}_{1}$ receptor immunoreactivity is found in many mouse retinal neurons, including rod and cone photoreceptors and ipRGCs. ${ }^{9,16}$ Fujieda et al's ${ }^{17}$ studies indicate the $\mathrm{MT}_{1}$ receptor is detectable in the GCs, ACs, IPL, and OPL in guinea pig retina. However, we'd better investigate the melatonin system both in the nocturnal animals and diurnal animals. In the human retina, the $\mathrm{MT}_{1}$ receptor transcript is immunoreactive in the INL, GCL, and in the IS of outer photoreceptors. ${ }^{11,18,19}$ Natesan and Cassone's ${ }^{12}$ work shows us the Mella receptor mRNA is primarily located in the IS of photoreceptors, inner part of the INL, and GCL in the chick retina. However, the pinpoint cellular localization of the Mella receptor at the protein level in the retina of diurnal animals remains unclear.

High-resolution structures of two subtypes of melatonin receptor are demonstrated recently using XFEL analysis. ${ }^{20}$ The research on circadian rhythm-related melatonin receptors
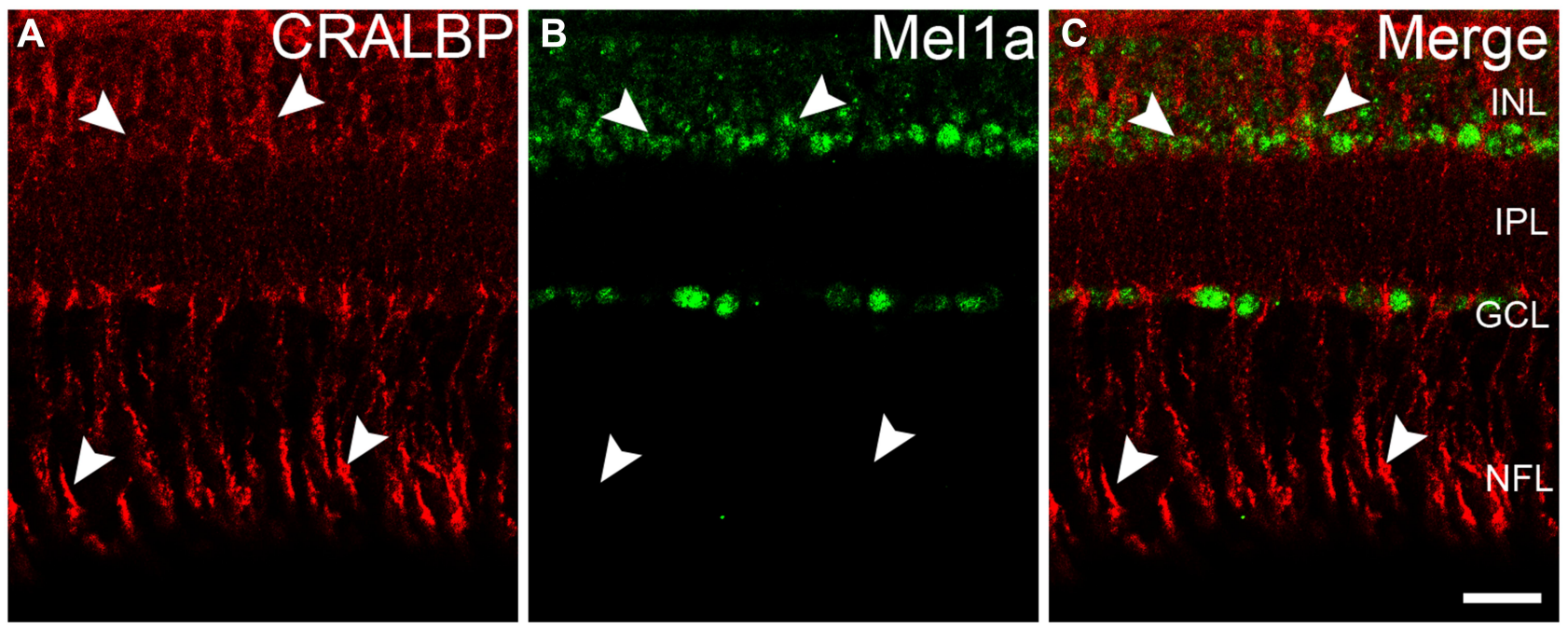

Figure 6 Mel la receptor immunoreactivity in glia Müller cells. (A-C) Representative data from retinal slice double-labeled by CRALBP and Mel la receptor. Mel la receptor immunostaining is observed in somata of CRALBP-positive Müller cells, while no obvious Mella receptor signal exists in processes of CRALBP-positive Müller cells. Representative neurons are marked by white arrowheads. 
will be beneficial to the study on pathogenesis of rhythmrelated discomfort or diseases and staying natural rhythms. So the studies on cellular localization of melatonin receptors should be placed on the agenda urgently. In the present work, we demonstrated the cellular localization of Mella receptor in pigeon retina by double immunofluorescence labeling. Mella receptor-positive signals were found in photoreceptors (OS of rods and red/green cones, somata of ipRGC), and somata of HCs, three subtypes of ACs (dopaminergic neurons, cholinergic neurons, glycinergic AII neurons) and GCs, while faint signals were observed in the IPL and no clearly Mella receptor signal was detected in the OS of blue cones, somata of BCs and OPL. At present, no research demonstrated retinal glia cells expressing melatonin receptor definitely, and our results have proved melatonin receptor Mella might be positive in Müller cells, especially in the somata. Regarding the issue of cytoplasmic staining of Mella receptor, we reported similar cases before, ${ }^{7,8}$ and the staining pattern appearing to localize to the cytoplasm, rather than to the membrane, is similar to those previous studies. ${ }^{21,22}$

Interestingly, the Mella receptor is expressed in red/ green cone and rod photoreceptors, but not in blue cones, which suggests melatonin may prefer to modulate the red/ green cone pathway and rod pathway via activating the Mella receptor. More, the expression of melatonin receptor in inhibitory neurons HCs and ACs, but not BCs, suggests a role of melatonin in the inhibitory neuromodulation pathway rather than in the excitatory neuromodulation pathway. According to our studies, the Mella receptor and Mellb receptor are co-expressed in rods, red/green cones, dopaminergic neurons, cholinergic neurons, glycinergic AII neurons, conventional ganglion cells, and intrinsically photosensitive retinal ganglion cells, while the two receptors seem to be negative in bipolar cells in pigeon retina. Localizational heterogeneity of the Mella receptor and Mellb receptor is mainly behaved in blue cones, horizontal cells, and Müller cells. Specifically, the Mella receptor prefers to be expressed in horizontal cells and Müller cells, when the Mellb receptor favors being localized in blue cones.

Corresponding to other diurnal animals, such as mammalian human and monkey, the $\mathrm{MT}_{1}$ receptor is expressed in photoreceptors, HCs, ACs, and GCs. That indicates no matter in mammalian or non-mammalian animals $\mathrm{MT}_{1}$ or Mella receptor owns same or similar cellular localization in diurnal animals. $^{11,18,19,23}$ However, it is noteworthy that $\mathrm{MT}_{1}$ or Mella receptors may be predominantly expressed in rods, cones, ACs, and GCs in the diurnal animals, ${ }^{11,12,18,19,23,24}$ while this receptor frequently appears in the IPL, OPL, and
GCs in the nocturnal animals. ${ }^{6,7,11-13,17-19}$ The different characters on localization of $\mathrm{MT}_{1}$ or Mella receptor may indeed contribute to the divergent circadian rhythms. How melatonin modulates these pathways differentially and how such modulation is integrated to modify retinal rhythmicity, or even circadian rhythm in the central nervous system will be an issue of great interest.

\section{Acknowledgments}

We gratefully acknowledge financial support from the Youth Fund of Shandong Academy of Sciences (No. 2019QN001, 2019QN005), Natural Science Foundation of Shandong Province (No. ZR2018PC013), International Science and Technology Cooperation Program of Shandong Academy of Sciences (No. 2019GHZD10), and National Key R\&D Program of China (No. 2018YFC1707300).

\section{Disclosure}

The authors report no conflicts of interest in this work.

\section{References}

1. Huang H, Wang Z, Weng SJ, Sun XH, Yang XL. Neuromodulatory role of melatonin in retinal information processing. Prog Retin Eye Res. 2013;32:64-87. doi:10.1016/j.preteyeres.2012.07.003

2. Cosci B, Longoni B, Marchiafava PL. Melatonin induces membrane conductance changes in isolated retinal rod receptor cells. Life Sci. 1997;60:1885-1889. doi:10.1016/S0024-3205(97)00150-1

3. Fischer TW, Zmijewski MA, Wortsman J, Slominski A. Melatonin maintains mitochondrial membrane potential and attenuates activation of initiator (casp-9) and effector caspases (casp-3/casp-7) and PARP in UVR-exposed HaCaT keratinocytes. $J$ Pineal Res. 2008;44:397-407. doi:10.1111/j.1600-079X.2007.00542.x

4. Yang XF, Miao Y, Ping Y, Wu HJ, Yang XL, Wang Z. Melatonin inhibits tetraethylammonium-sensitive potassium channels of rod ON type bipolar cells via MT2 receptors in rat retina. Neuroscience. 2011;173:19-29. doi:10.1016/j.neuroscience.2010.11.028

5. Tosini G, Baba K, Hwang CK, Iuvone PM. Melatonin: an underappreciated player in retinal physiology and pathophysiology. Exp Eye Res. 2012;103:82-89. doi:10.1016/j.exer.2012.08.009

6. Pack W, Hill DD, Wong KY. Melatonin modulates M4-type ganglioncell photoreceptors. Neuroscience. 2015;303:178-188. doi:10.1016/j. neuroscience.2015.06.046

7. Sheng WL, Chen WY, Yang XL, Zhong YM, Weng SJ. Co-expression of two subtypes of melatonin receptor on rat M1-type intrinsically photosensitive retinal ganglion cells. PLoS One. 2015;10: e0117967. doi:10.1371/journal.pone.0117967

8. Sheng W, Jin M, Pan G, et al. Cellular localization of melatonin receptor Mel1b in pigeon retina. Neuropeptides. 2019;78:101974. doi:10.1016/j.npep.2019.101974

9. Sengupta A, Baba K, Mazzoni F, et al. Localization of melatonin receptor 1 in mouse retina and its role in the circadian regulation of the electroretinogram and dopamine levels. PLoS One. 2011;6: e24483. doi: 10.1371 journal.pone.0024483

10. Ikegami T, Motohashi E, Doi H, Hattori A, Ando H. Synchronized diurnal and circadian expressions of four subtypes of melatonin receptor genes in the diencephalon of a puffer fish with lunar-related spawning cycles. Neurosci Lett. 2009;462:58-63. doi:10.1016/j. neulet.2009.06.076 
11. Scher J, Wankiewicz E, Brown GM, Fujieda H. AII amacrine cells express the MT1 melatonin receptor in human and macaque retina. Exp Eye Res. 2003;77:375-382. doi:10.1016/S0014-4835(03)00123-4

12. Natesan AK, Cassone VM. Melatonin receptor mRNA localization and rhythmicity in the retina of the domestic chick, Gallus domesticus. Vis Neurosci. 2002;19:265-274. doi:10.1017/ S0952523802192042

13. Fujieda H, Hamadanizadeh SA, Wankiewicz E, Pang SF, Brown GM. Expression of $\mathrm{mt} 1$ melatonin receptor in rat retina: evidence for multiple cell targets for melatonin. Neuroscience. 1999;93:793-799. doi:10.1016/S0306-4522(99)00111-6

14. Dubocovich ML, Markowska M. Functional MT1 and MT2 melatonin receptors in mammals. Endocrine. 2005;27:101-110. doi:10.1385/ENDO:27:2:101

15. Pandi-Perumal SR, Trakht I, Srinivasan V, et al. Physiological effects of melatonin: role of melatonin receptors and signal transduction pathways. Prog Neurobiol. 2008;85:335-353. doi:10.1016/j. pneurobio.2008.04.001

16. Baba K, Pozdeyev N, Mazzoni F, et al. Melatonin modulates visual function and cell viability in the mouse retina via the MT1 melatonin receptor. Proc Natl Acad Sci U S A. 2009;106:15043-15048. doi:10.1073/pnas.0904400106

17. Fujieda H, Scher J, Hamadanizadeh SA, Wankiewicz E, Pang SF, Brown GM. Dopaminergic and GABAergic amacrine cells are direct targets of melatonin: immunocytochemical study of mt1 melatonin receptor in guinea pig retina. Vis Neurosci. 2000;17:63-70. doi: $10.1017 /$ S0952523800171068
18. Savaskan E, Wirz-Justice A, Olivieri G, et al. Distribution of melatonin MT1 receptor immunoreactivity in human retina. J Histochem Cytochem. 2002;50:519-526. doi:10.1177/002215540205000408

19. Scher J, Wankiewicz E, Brown GM, Fujieda H. MT(1) melatonin receptor in the human retina: expression and localization. Invest Ophthalmol Vis Sci. 2002;43:889-897.

20. Stauch B, Johansson LC, McCorvy JD, et al. Structural basis of ligand recognition at the human MT1 melatonin receptor. Nature. 2019;569:284-288. doi:10.1038/s41586-019-1141-3

21. Wu YH, Ursinus J, Zhou JN, et al. Alterations of melatonin receptors MT1 and MT2 in the hypothalamic suprachiasmatic nucleus during depression. J Affect Disord. 2013;148:357-367. doi:10.1016/j. jad.2012.12.025

22. Hunt AE, Al-Ghoul WM, Gillette MU, Dubocovich ML. Activation of MT(2) melatonin receptors in rat suprachiasmatic nucleus phase advances the circadian clock. Am J Physiol Cell Physiol. 2001;280: C110-C118. doi:10.1152/ajpcell.2001.280.1.C110

23. Meyer P, Pache M, Loeffler KU, et al. Melatonin MT-1-receptor immunoreactivity in the human eye. $\mathrm{Br} J$ Ophthalmol. 2002;86:1053-1057. doi:10.1136/bjo.86.9.1053

24. Wu YH, Zhou JN, Balesar R, et al. Distribution of MT1 melatonin receptor immunoreactivity in the human hypothalamus and pituitary gland: colocalization of MT1 with vasopressin, oxytocin, and corticotropin-releasing hormone. J Comp Neurol. 2006;499:897-910. doi:10.1002/cne.21152

\section{Publish your work in this journal}

Nature and Science of Sleep is an international, peer-reviewed, open access journal covering all aspects of sleep science and sleep medicine, including the neurophysiology and functions of sleep, the genetics of sleep, sleep and society, biological rhythms, dreaming, sleep disorders and therapy, and strategies to optimize healthy sleep.
The manuscript management system is completely online and includes a very quick and fair peer-review system, which is all easy to use. Visit http://www.dovepress.com/testimonials.php to read real quotes from published authors. 